\title{
Editorial
}

\section{Non-Abelian Gauge Symmetries beyond the Standard Model}

\author{
Hoang Ngoc Long, ${ }^{1}$ Vicente Pleitez, ${ }^{2}$ \\ Marc Sher, ${ }^{3}$ and Masaki Yasue ${ }^{4}$ \\ ${ }^{1}$ Institute of Physics, Vietnamese Academy of Science and Technology, 10 Dao Tan, Ba Dinh, \\ Hanoi, Vietnam \\ ${ }^{2}$ Instituto de Fisica Teorica, Universidade Estadual Paulista, Rua DR. Bento Terobalo Ferraz 271, \\ Bloco II Barra Funda, 01140-070 São Paulo, SP, Brazil \\ ${ }^{3}$ Particle Theory Group, Department of Physics, College of William and Mary, Williamsburg, \\ VA 23187, USA \\ ${ }^{4}$ Department of Physics, Tokai University, 4-1-1 KitaKaname, Hiratsuka, Kanagawa 259-1292, Japan \\ Correspondence should be addressed to Hoang Ngoc Long, hnlong@iop.vast.ac.vn
}

Received 6 February 2012; Accepted 6 February 2012

Copyright (c) 2012 Hoang Ngoc Long et al. This is an open access article distributed under the Creative Commons Attribution License, which permits unrestricted use, distribution, and reproduction in any medium, provided the original work is properly cited.

The recent groundbreaking discovery of nonzero neutrino masses and oscillations is strong evidence of physics beyond the SM. The gauge symmetry of the SM and those of many extensions fix only the structure of gauge bosons. The fermions and Higgs representations have to be chosen somewhat arbitrarily. Thus, the models with the non-Abelian SM gauge symmetries are the main subject in this special issue. The authors have focused on models based on the $S U(3)_{C} \otimes S U(3)_{L} \otimes U(1)_{X}$ (3-3-1) gauge group, left-right symmetric model, and models with discrete symmetries. The gauge-Higgs unification based on the space-time extension has been also discussed in the issue.

The paper "Mass mixing effect and oblique radiative corrections in extended $S U(2)_{R} \otimes$ $S U(2)_{L} \otimes U(1)$ effective theory" by Y. Zhang analyzes the properties of electroweak chiral effective Lagrangian with an extended $S U(2)_{R}$ gauge group. The non-Abelian $S U(2)_{R}$ contains sufficient complexity to incorporate interesting issues related to spontaneous parity violation and precise electroweak observables. The author discusses all possible mass-mixing terms and calculates the exact physical mass eigenvalues by diagonalization of mixing matrix without any approximations. The contributions to oblique radiative corrections parameters STU from $S U(2)_{R}$ fields are also presented.

In the paper "Sources of FCNC in $S U(3)_{C} \otimes S U(3)_{L} \otimes U(1)_{X}$ models" by J. M. Cabarcas et al., the authors explore the possible sources of flavor changing neutral currents and lepton flavor violation at tree level, in the 3-3-1 models. In the paper "Non-standard neutrinos interactions in a 3-3-1 model with minimum Higgs sector" by M. M. Jaime and P. C. de Holanda, 
the nonstandard neutrino interactions in the economical 3-3-1 model is presented. The limit on new gauge bosons is obtained.

The paper "Gauge boson mixing in the 3-3-1 models with discrete symmetries" by Dong Phung et al. deals with the mixing among gauge bosons in the 3-3-1 model with the discrete symmetries. The authors have shown that the neutrino tribimaximal mixing leads to the CPT violation. In the paper "Radiatively generated leptogenesis in $S_{4}$ flavor symmetry models" by T. P. Nguyen and D. Phung radiatively generated leptogenesis in the $S_{4}$ flavor symmetry models is presented. The authors have found a link between leptogenesis and amplitude of neutrinoless double beta decay $\left|m_{e e}\right|$ through a high-energy CP phase $\phi$.

The paper "Gauge-Higgs unification models in six dimensions with $S_{2} / Z_{2}$ extra space and GUT gauge symmetry" by C.-W. Chiang et al. reviews gauge-Higgs unification models in six dimensions with $S_{2} / Z_{2}$ extra space and GUT gauge symmetry. It presents two scenarios for constructing a four-dimensional theory from the six-dimensional model, which leads to an SM-like gauge theory with the $S U(3) \otimes S U(2)_{L} \otimes U(1)_{Y}\left(\otimes U(1)_{2}\right)$ symmetry and the SM fermions in four dimensions. The gauge boson and Higgs boson masses are obtained.

Hoang Ngoc Long Vicente Pleitez

Marc Sher Masaki Yasue 

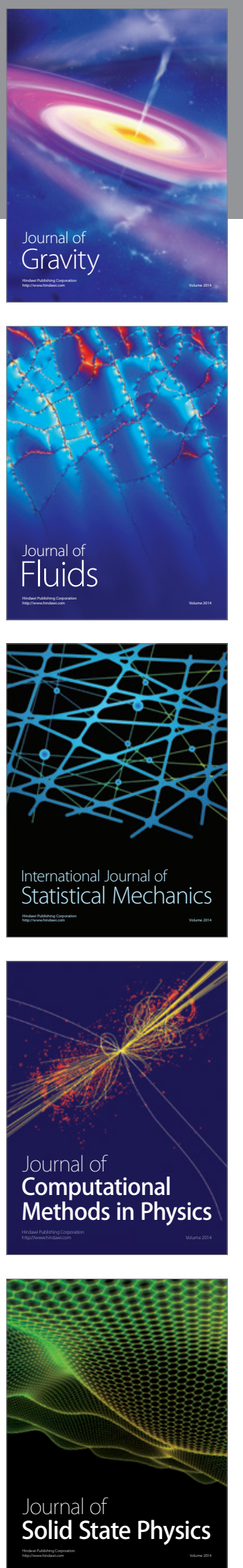

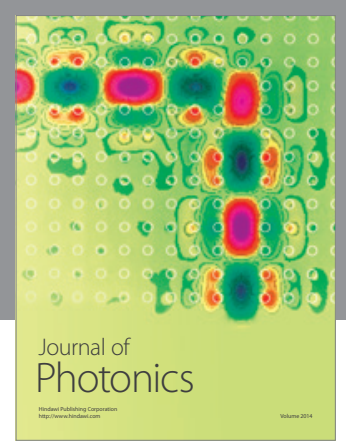

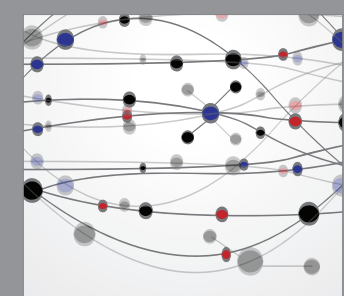

The Scientific World Journal
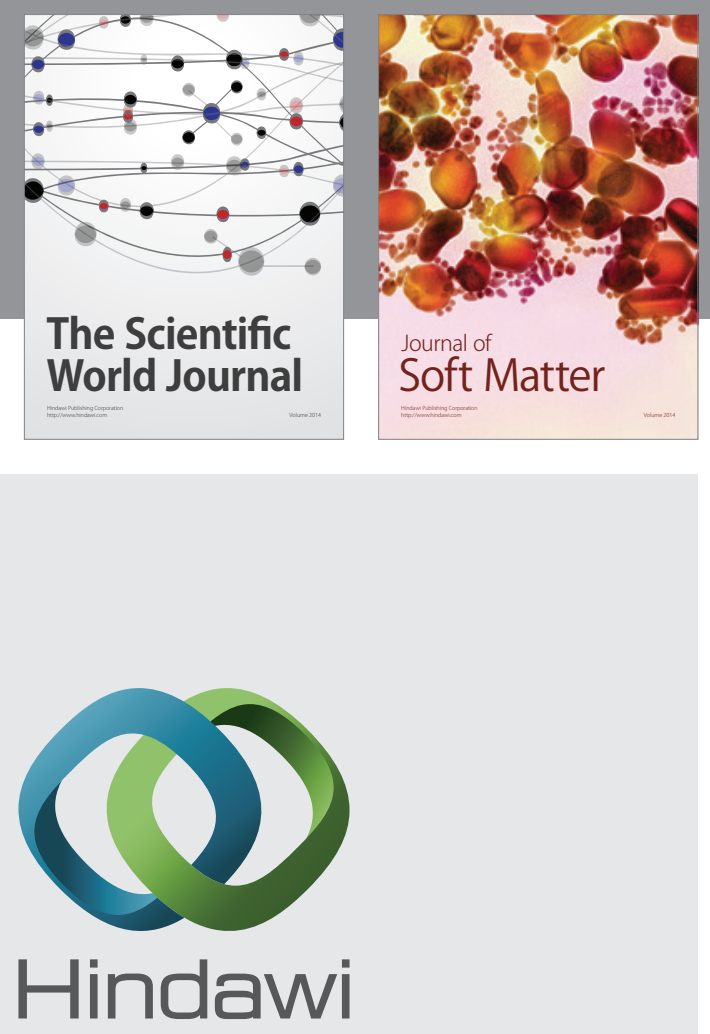

Submit your manuscripts at

http://www.hindawi.com
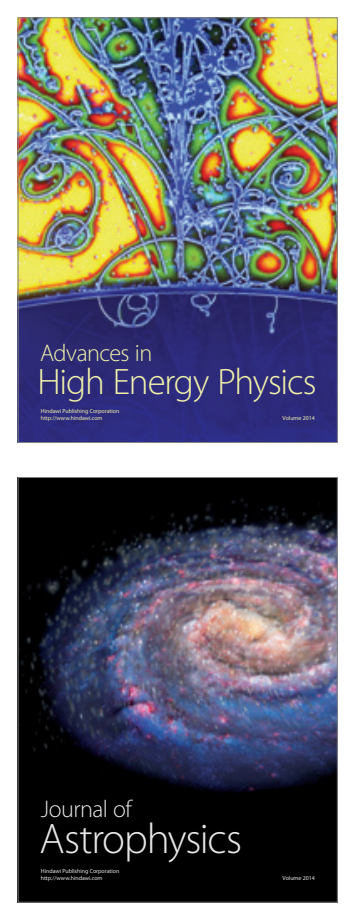
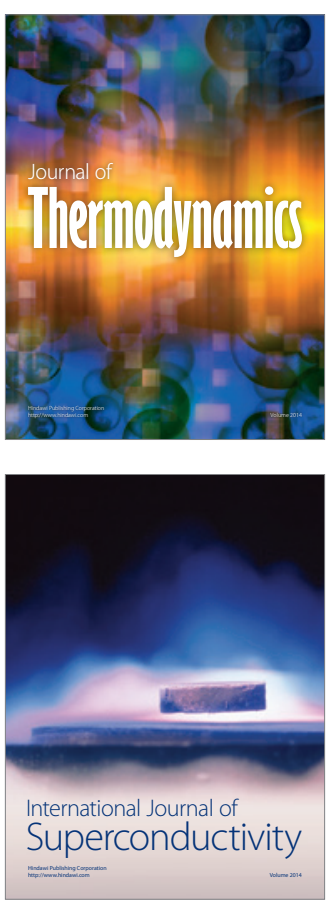
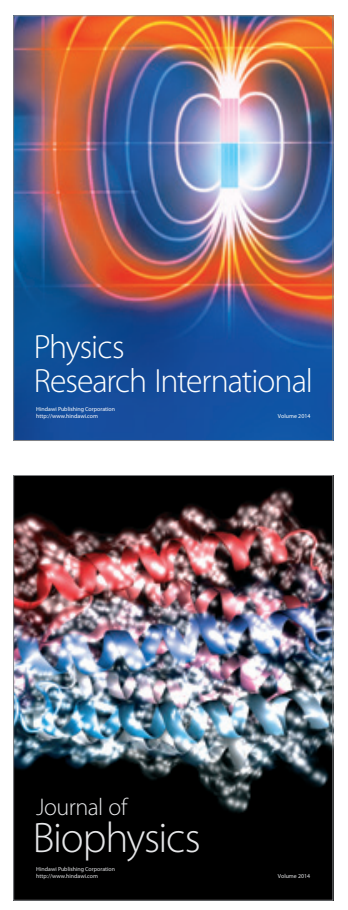
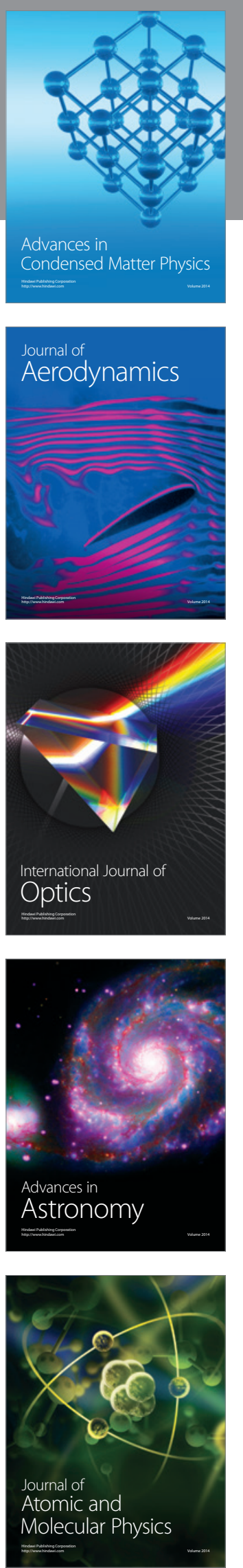